\title{
Potencial de deriva da mistura de 2,4-D com glyphosate ${ }^{1}$
}

\author{
Potential for drifting of mix 2,4-D + glyphosate
}

\begin{abstract}
Marco Antonio Gandolfo ${ }^{2}$; Eder Dias Moraes ${ }^{3}$; Ulisses Delvaz Gandolfo ${ }^{4}$; Jethro Barros Osipe ${ }^{5}$; Eurípedes Bomfim Rodrigues ${ }^{2}$; Robinson Osipe ${ }^{2}$
\end{abstract}

Resumo - A aplicação da mistura dos herbicidas glyphosate com 2,4-D passou a ter grande importância com a adoção do sistema de semeadura direta, no início dos anos 90, e com os constantes relatos de biótipos de plantas daninhas resistentes ao herbicida glyphosate. No entanto, estudos referentes à deriva desta mistura ainda são escassos. O objetivo deste trabalho foi avaliar o potencial de deriva dos herbicidas glyphosate e 2,4-D, associados ou não a adjuvante, pulverizados em túnel de vento. O delineamento experimental considerado foi inteiramente ao acaso, arranjados em um esquema fatorial $5 \times 3$, com quatro repetições, sendo cinco caldas e três distâncias de coleta (5, 10 e 15 metros). As caldas testadas foram: glyphosate, 2,4-D, 2,4D+glyphosate e 2,4-D+glyphosate+adjuvante, além de uma testemunha na qual foi pulverizada água. A aplicação dos produtos ocorreu em um túnel com $20 \mathrm{~m}$ de comprimento e secção transversal de $4 \mathrm{~m}^{2}$, com velocidade do vento de $2,0 \mathrm{~m} \cdot \mathrm{s}^{-1}$. Os pontos de coleta foram instalados a 5,10 e $15 \mathrm{~m}$ de distância da barra de pulverização e nestes pontos a cada $20 \mathrm{~cm}$ de altura em relação ao piso do túnel até $1,0 \mathrm{~m}$ de altura. Os valores médios obtidos nas coletas foram submetidos à análise de variância, e suas médias foram comparadas pelo teste Tukey a 5\% de probabilidade. Observou-se que a aplicação dos herbicidas associados produz mais deriva do que quando aplicados de forma isolada, principalmente a 5 e a 10 metros de distância da barra de pulverização. A deriva de cada herbicida aplicado isoladamente não apresentou diferenças estatísticas. Quando se adicionou adjuvante a calda associada houve uma redução de $23 \%$ na deriva a $5 \mathrm{~m}$ e $6 \%$ a $10 \mathrm{~m}$.

Palavras-chaves: herbicidas, tecnologia de aplicação, túnel de vento

\begin{abstract}
The application of the mixture of glyphosate with 2,4-d took on great importance with high adoption of no-tillage system in the early 90s, and with the constant reports of weed biotypes resistant to glyphosate. However, studies on the drift of this mixture are still scarce. The aim of this study was to evaluate the potential drift of these herbicides, with or without the adjuvant, applied in wind tunnel. The experimental design was completely randomized considered, arranged in a factorial design $5 \times 3$, with four replications, and five tails and three collection distances $(5,10$ and 15 meters). The spray tested were: glyphosate, 2,4-D, 2,4D+glyphosate, glyphosate+2,4-D+adjuvant, and a control in which water was applied. The collection points were installed at 5,10 and 15 meters and in this points each $20 \mathrm{~cm}$ of height in relation of tunnel ground until $1,0 \mathrm{~m}$ of height. The application of the products took place in a

\footnotetext{
${ }^{1}$ Recebido para publicação em 29/10/2012 e aceito em 19/12/2012.

${ }^{2}$ Professor Doutor em Agronomia pela Universidade Estadual do Norte do Paraná - Campus Luiz Meneghel (UENP-CLM). Rodovia BR 369, km 54 - gandolfo@ uenp.edu.br;

${ }^{3}$ Eng. Agrônomo pela Universidade Estadual do Norte do Paraná;

${ }^{4}$ Mestrando em Agronomia pela Universidade Estadual Paulista (UNESP-FCA);

${ }^{5}$ Doutorando em Agronomia pela Universidade Estadual de Maringá (UEM).
} 
wind tunnel with $20 \mathrm{~m}$ length and cross-sectional $4 \mathrm{~m}^{2}$ with speed wind of $2.0 \mathrm{~m} \mathrm{~s}^{-1}$, The mean values obtained in the samples were subjected to analysis of variance, and their means were compared by Tukey test at $5 \%$ probability. It was observed that herbicide application associated produces more drift than when applied alone mainly to 5 and 10 meters of distance to sprayer boom. The drift of each herbicide applied alone did not differ. When added to the adjuvant mixture glyphosate plus 2,4-D, there was a $23 \%$ reduction in drift at $5 \mathrm{~m}$ and $6 \mathrm{~m}$ to $10 \%$.

Keywords: herbicides, application technology, wind tunnel

\section{Introdução}

Como grande parte das áreas agrícolas do Brasil faz uso do sistema de semeadura direta, há uma enorme necessidade da utilização do controle químico das plantas daninhas (Costa et al., 2012). Por não ser possível o revolvimento do solo nesse sistema, a necessidade de herbicidas dessecantes no manejo da comunidade infestante, antecedendo a semeadura das culturas, é fundamental (Oliveira Jr. et al, 2007). Dentre os herbicidas mais utilizados estão o 2,4-D e o glyphosate, os quais se destacam pela sua reconhecida eficiência e relação custo benefício.

Para o controle de biótipos de Conyza spp. (buva) resistentes ao glyphosate, a aplicação da mistura do herbicida glyphosate com o 2,4-D é um excelente ferramenta de manejo, principalmente quando as plantas de buva estão com alturas inferiores a $11 \mathrm{~cm}$, (Oliveira Neto et al, 2010a, Oliveira Neto et al., 2010b). Flint \& Barret et al. (1989) afirmam que a mistura entre os herbicidas apresentam efeitos aditivos ou sinergísticos para o controle de Convolvulus arvensis.

O herbicida 2,4-D é registrado no Brasil para o controle de dicotiledôneas em pósemergência em café, cana-de-açúcar, cereais, manejo em plantio direto, gramados, pastagens, canais, açudes, represas, espelhos d'água e áreas não cultivadas. É de persistência curta no solo, o que permite a semeadura de culturas que lhe são suscetíveis duas semanas depois da aplicação. É formulado em sais de amina e éster, sendo estes mais agressivos tanto para as plantas daninhas como para as culturas; são mais voláteis quanto menor o número de carbonos contidos na fração álcool que constitui sua molécula (Rodrigues \& Almeida, 2011).

Deve-se ter cuidado especial na aplicação do 2,4-D, não o utilizando em áreas próximas a culturas suscetíveis e em condições inadequadas de vento, em função da deriva (desvio do produto aplicado, fazendo com que este não atinja o alvo da aplicação). A ocorrência de deriva acidental na aplicação de herbicidas é considerada um dos sérios problemas da agricultura, por reduzir a eficiência da aplicação, além de colocar em risco as culturas instaladas em áreas circunvizinhas (Tuffi Santos et al., 2006a, b; Yamashita et al., 2006, Yamashita \& Guimarães, 2006).

Gazziero et al. (2000) destacam os efeitos prejudiciais causados pela deriva de 2,4-D nos cultivos de algodão, uva e hortaliças. Oliveira Jr. et al. (2007), Constantin et al. (2007a), Constantin et al. (2007b) constataram efeitos prejudiciais causados por deriva simulada (subdoses) desse herbicida, sobre as culturas do uva, algodão e fumo, respectivamente.

No entanto, ressalta-se que o potencial do 2,4-D em gerar deriva é compatível com os de outros herbicidas presentes no mercado, ganhando destaque, neste cenário, devido aos sintomas causados pela deriva serem bastantes característicos em doses mínimas, criando a falsa impressão de uma deriva maior para esse produto (Kissmann, 2001).

A utilização de técnicas corretas de aplicação pode efetivamente reduzir o risco ou a quantidade de deriva produzida nas aplicações de agroquímicos. Estudos são necessários para que se busquem técnicas mais 
seguras para aplicações de misturas, especificamente para 2,4-D e o glyphosate. Sendo assim, este trabalho teve por objetivo avaliar a deriva proporcionada pela mistura de 2,4-D com glyphosate, associada ou não a adjuvante, aplicado com pontas de pulverização de gotas finas.

\section{Material e Métodos}

$\mathrm{O}$ ensaio foi realizado nas instalações do Núcleo de Investigação em Tecnologia de aplicação de Agroquímicos e Máquinas Agrícolas - NITEC da Universidade Estadual do Norte do Paraná - Campus Luiz Meneghel - Bandeirantes, PR (latitude $23,1^{\circ} \mathrm{S}$, longitude $54,4^{\circ} \mathrm{W}$ e altitude de $\left.440 \mathrm{~m}\right)$.

A condução do ensaio ocorreu em um túnel de vento de secção quadrada de $2,0 \mathrm{~m}$, com $20 \mathrm{~m}$ de comprimento, equipado com barra de pulverização de 1,0 metro de largura transversal ao comprimento do túnel. $\mathrm{Na}$ entrada do túnel foi instalado um ventilador axial de $90 \mathrm{~mm}$ de diâmetro, movida pela tdp (tomada de potência) de um trator com motor de $65 \mathrm{~kW}$ (quilowatts) e rotação de $1200 \mathrm{rpm}$ (rotações por minuto).

A velocidade do vento para os ensaios foi de $2,0 \mathrm{~m} \mathrm{~s}^{-1}$ medida a cinco metros da barra de pulverização. A barra foi posicionada a dois metros de distância, em relação aos defletores da colmeia do difusor da ventilação e nela foram instalados três bicos de pulverização espaçados a $50 \mathrm{~cm}$ e altura de $60 \mathrm{~cm}$ em relação ao piso do túnel. A pressão do circuito hidráulico foi de $276 \mathrm{kPa}$ (40 psi), e a taxa de aplicação obtida foi de $100 \mathrm{~L} \mathrm{ha}^{-1}$.

Foi utilizada a ponta de pulverização de jato plano comum modelo AXI 110015. O circuito hidráulico constou de uma bomba de pistão movida por um motor elétrico de $3,0 \mathrm{~kW}$ com controlador de pressão mecânico. $\mathrm{O}$ marcador utilizado foi o $\mathrm{NaCl}$ na concentração de $10 \% \mathrm{v}^{-1}{ }^{-1}$ segundo metodologia descrita por Abi Saab (2000).

$\mathrm{O}$ delineamento experimental considerado foi inteiramente ao acaso, arranjados em um esquema fatorial $5 \times 3$, sendo cinco caldas e três distâncias de coleta (5, 10 e 15 metros).

As cinco caldas foram: os herbicidas glyphosate e 2,4-diclorofenoxiacético (2,4-D) isoladamente, e em mistura; a mistura dos herbicidas e um adjuvante redutor de deriva na concentração de $1,5 \% \mathrm{v} \mathrm{v}^{-1}$ (fosfatidilcoline + ácido propiônico) e testemunha composta apenas por $\mathrm{NaCl}$, conforme descrito na Tabela 1. O experimento foi conduzido com quatro repetições.

Tabela 1. Descrição das caldas utilizadas para o experimento. Bandeirantes, PR.

\begin{tabular}{ll}
\hline Tratamentos & Concentração $\left(\mathbf{v ~ v}^{-1}\right) /$ Doses* $^{*}\left(\mathbf{e} . \mathbf{h a}^{-1}\right)$ \\
\hline $\mathrm{NaCl}$ (testemunha) & $10 \%$ \\
$\mathrm{NaCl}+\mathrm{Glyphosate}$ & $10 \%+720 \mathrm{~g}$ \\
$\mathrm{NaCl}+2,4-\mathrm{D}$ & $10 \%+670 \mathrm{~g}$ \\
$\mathrm{NaCl}+\mathrm{Glyphosate}+2,4-\mathrm{D}$ & $10 \%+720 \mathrm{~g}+670 \mathrm{~g}$ \\
$\mathrm{NaCl}+$ Adjuvante + Glyphosate+2,4-D & $10 \%+1,5 \%+720 \mathrm{~g}+670 \mathrm{~g}$ \\
\hline
\end{tabular}

* 2,4-D - DMA 806BR; glyphosate - Roundup Original; Adjuvante - fosfatidilcoline + ácido propiônico

Os coletores foram fios de polietileno de $2 \mathrm{~mm}$ de diâmetro com $30 \mathrm{~cm}$ de comprimento, fixados em três suportes de metal distando 5, 10 e 15 metros da barra de pulverização. Em cada suporte de metal foram instalados cinco fios posicionados a 20,40,60, 80 e $100 \mathrm{~cm}$ do piso do túnel. As médias dos depósitos de marcador nos cinco fios nas diferentes alturas representaram os valores de cada distância testada. Após as pulverizações os fios eram colocados em um tubo de PVC de $0,50 \mathrm{~m}$ de comprimento com $50 \mathrm{~cm}^{3}$ de água deionizada para lavagem. 
Para as leituras de condutividade elétrica, foi utilizado um condutivímetro digital com precisão de $1,0 \mu \mathrm{S}$ e escala máxima de $1999 \mu \mathrm{S}$ que foi mergulhado na água de lavagem dos tubos para medição da condutividade elétrica. Esta medição permitiu a comparação dos valores de condutividade elétrica em cada distância pelo volume de líquido deslocado pelo vento durante as pulverizações, sendo estes valores relacionados à deriva obtida em cada tratamento. Foram realizadas também avaliações de condutividade elétrica em dez tubos com dez fios sem uso, para correção da condutividade presente no próprio alvo, assegurando assim a leitura corrigida das amostras.
Os valores médios de condutividade foram submetidos à análise de variância pelo teste $\mathrm{F}$, e suas médias comparadas pelo teste Tukey a 5\% de probabilidade para o fator qualitativo.

\section{Resultados e Discussão}

Os resultados obtidos na análise de variância mostraram que a interação entre caldas e distâncias foi significativa pelo teste $\mathrm{F}$ a $5 \%$ de probabilidade (Tabela 2), sendo efetuado, portanto, o desdobramento das interações.

Os resultados médios de deriva referentes às diferentes caldas em função das distâncias são apresentados na Figura 1.

Tabela 2. Análise de variância do ensaio. Bandeirantes, PR.

\begin{tabular}{lcccc}
\hline F.V. & G.L. & S.Q. & Q.M. & Fc \\
\hline Caldas & 4 & 2562,81 & 640,70 & $31,20^{* *}$ \\
Distâncias & 2 & 8101,78 & 4050,89 & $197,25^{* *}$ \\
Caldas * Distâncias & 8 & 791,18 & 98,90 & $4,82^{* *}$ \\
Resíduo & 45 & 924,14 & 20,54 & \\
\hline Total & 59 & 12379,91 & & \\
\hline
\end{tabular}

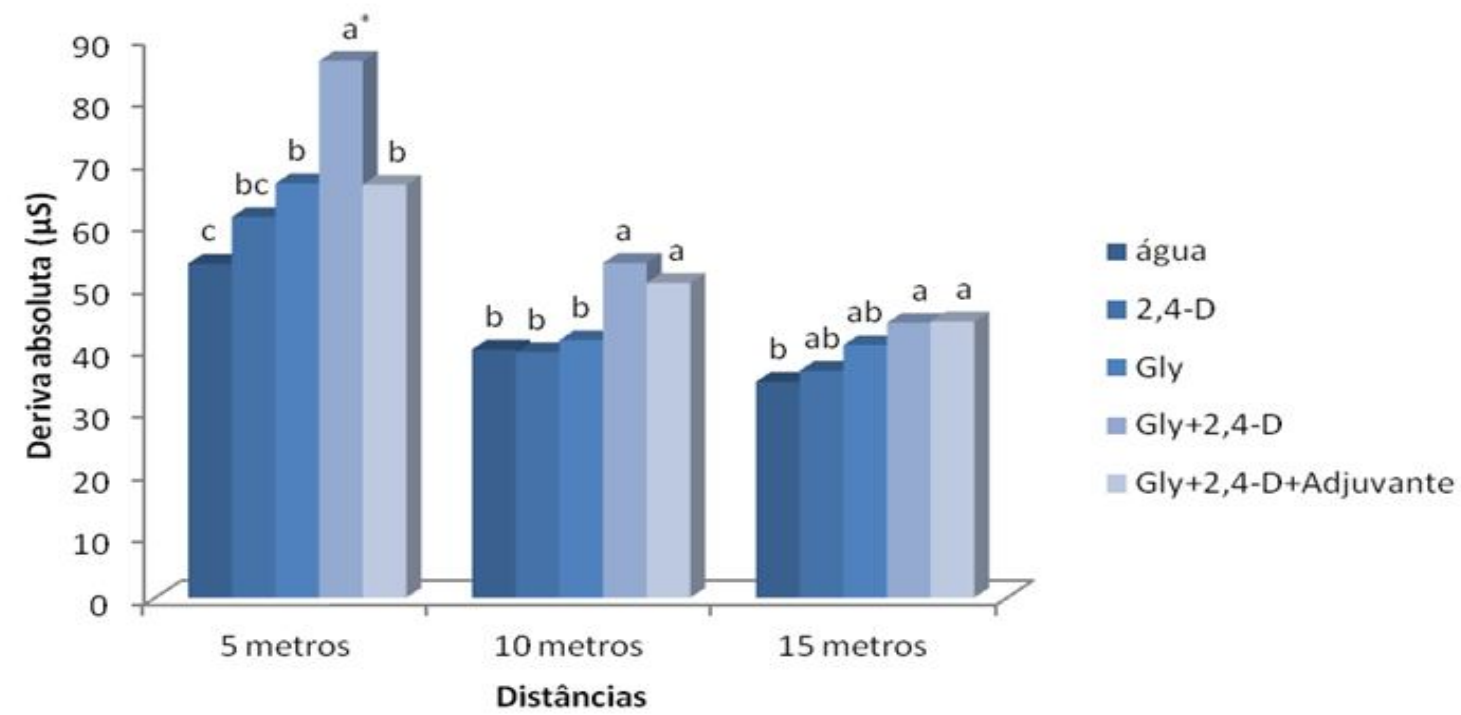

*Médias seguidas da mesma letra, em cada distância, não diferem entre si pelo teste Tukey ao nível de 5\% de probabilidade.

Figura 1. Médias da deriva absoluta coletada $(\mu \mathrm{S})$ de cada tipo de calda em função da distância de coleta. (C.V. $=8,95 \%$; DMS=9,08). Bandeirantes, PR. 
A Figura 1 revela que na distância de 5 metros, a deriva absoluta coletada da mistura glyphosate + 2,4-D foi superior à deriva identificada nos demais tratamentos. No entanto, quando se utilizou o adjuvante fosfatidilcoline + ácido propiônico associado aos herbicidas houve uma redução de $23 \%$ na deriva coletada, não havendo diferença das aplicações isoladas dos herbicidas. Uma vez que a mistura dos herbicidas potencializa a deriva coletada e que sua redução pode se dar pela adição de um adjuvante, a colocação deles na calda ou a aplicação dos herbicidas de forma isolada pode reduzir os riscos de contaminação do meio. Os efeitos fitotóxicos do herbicida 2,4-D em culturas sensíveis ocorrem com doses relativamente baixas, sendo da ordem de $1.10^{-3}$ em relação àquelas recomendadas para o controle, de acordo com resultados de estudos obtidos Hemphill \& Montgomery (1981) e Gilreath et al. (2001).

Entre as aplicações isoladas de glyphosate e 2,4-D, não existiram diferenças de deriva. Aos 10 metros de distância, as aplicações das misturas glyphosate+2,4-D, independente da presença ou ausência do adjuvante, mostraram-se com maior potencial de deriva que os demais tratamentos. $\mathrm{O}$ tratamento no qual se utilizou o adjuvante, apesar de não diferir estatisticamente daquele no qual se aplicou glyphosate+2,4-D, apresentou valor de deriva $6 \%$ inferior. Antuniassi (2006) revela que as características de uma pulverização podem ser alteradas significativamente com a adição de adjuvantes. Thebaldi et al. (2009), ao estudarem o efeito da adição de adjuvantes na redução de deriva, demonstram que adjuvantes são eficientes para esta finalidade.

Novamente, quando se comparam as aplicações isoladas de 2,4-D e glyphosate, não é possível verificar diferenças entre os valores de deriva coletada.

Já para a distância de 15 metros, não é possível notar diferenças significativas entre as aplicações isoladas ou em mistura. As únicas diferenças observadas são entre os tratamentos com a mistura glyphosate+2,4-D e glyphosate+2,4-D+adjuvante com o tratamento testemunha, com a aplicação dos herbicidas apresentando maiores valores de deriva.

Os resultados médios de deriva em função das distâncias são apresentados na Figura 2.

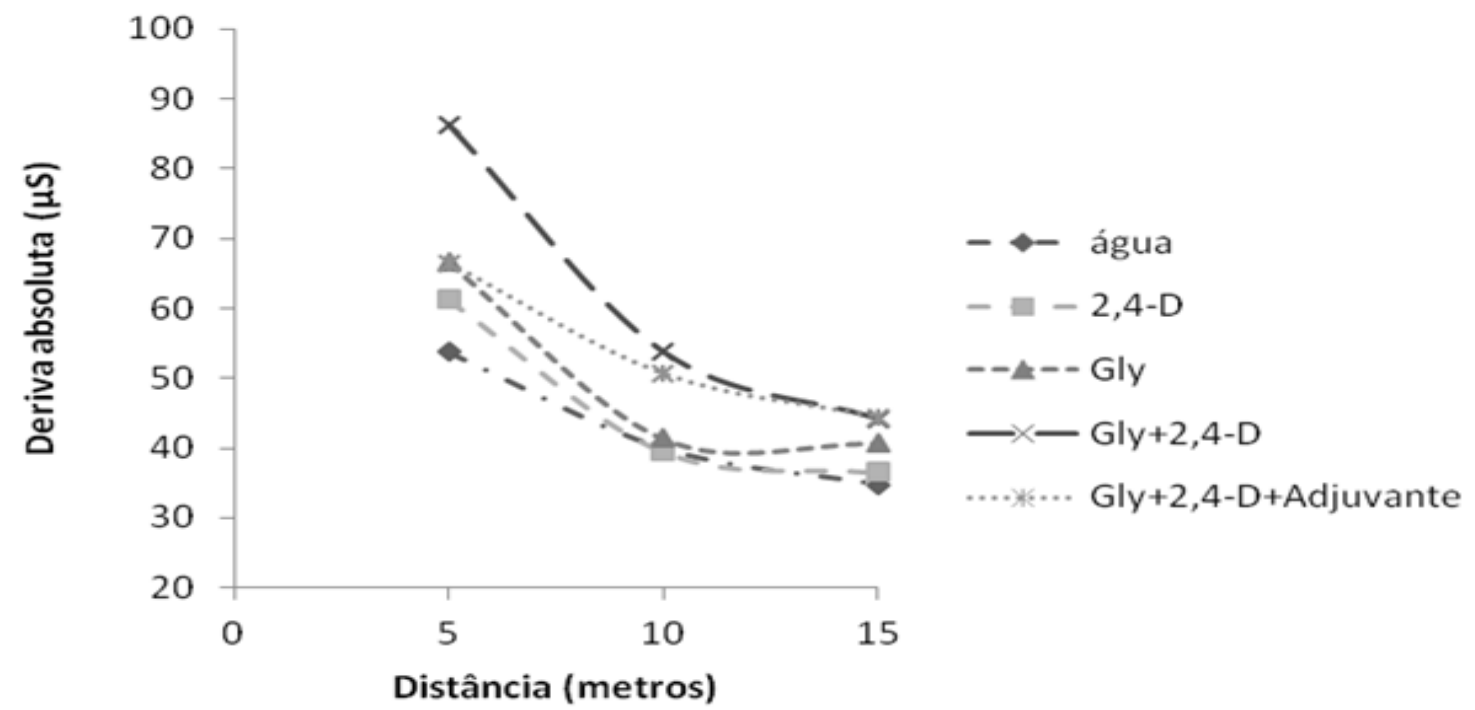

Figura 2. Médias da deriva absoluta $(\mu \mathrm{S})$ de cada distância de coleta calda em função do tipo de calda. Bandeirantes, PR. 
Percebe-se que, independente da calda utilizada, o potencial de deriva a 5 metros de distância é sempre superior às demais (10 e 15 metros de distância). Costa et al. (2012) observaram quantidades decrescentes de depósito de calda, principalmente até a distância de 20 metros, quando testaram o potencial de deriva da mistura glyphosate $+2,4-$ D. Trabalho de Nuyttens et al. (2006) corroboram também com os resultados apresentados, por mostrarem que, a medida em que se afasta da barra de pulverização, o potencial de deriva tende a ser menor.

A tendência de diminuição da deriva também ocorre de 10 para 15 metros, com exceção da calda formada por glyphosate apenas. Neste caso, a deriva observada foi muito próxima nas duas distâncias de coleta. Na calda formada por 2,4-D+glyphosate, a redução da deriva de 10 para 15 metros foi a mais acentuada.

Os resultados demonstram que quando a cultura vizinha está até 5 metros de distância do local de aplicação, existe um grande risco de ocorrer problemas de deriva, principalmente se as culturas vizinhas foram sensíveis ao herbicida aplicado. Entretanto, quando a distância é superior a $15 \mathrm{~m}$, o risco de deriva diminui.

\section{Conclusões}

A aplicação dos herbicidas glyphosate e 2,4-D de forma isolada produz menos deriva coletada até $10 \mathrm{~m}$ de distância da barra de pulverização do que quando em mistura.

A mistura dos herbicidas associados ao adjuvante fosfatidilcoline + ácido propiônico reduz a deriva coletada até 5 metros de distância do ponto de pulverização.

Entre os herbicidas aplicados isoladamente, não há diferenças nos níveis de deriva coletada.

Há uma tendência do decrescimento da deriva com o aumento da distância de coleta.

\section{Referências}

ABI SAAB, O. J. G. Avaliação da cobertura e depósitos de agrotóxicos em videiras com o uso de diferentes técnicas de aplicação e condições operacionais. 2000. 84f. Tese (Doutorado em Agronomia) - Faculdade de Ciências Agronômicas, Universidade Estadual Paulista, Botucatu, 2000.

ANTUNIASSI, U. R. Tecnologia de aplicação de defensivos. Revista Plantio Direto, v.15, n.4, p. 17-22, 2006.

CONSTANTIN, J. et al. Efeito de subdoses de 2,4-D na produtividade de algodão e suscetibilidade da cultura em função do seu estádio de desenvolvimento. Engenharia Agrícola, v.27, n.spe, p.24-29, 2007a.

CONSTANTIN, J. et al. Efeito de subdoses de 2,4-D na produtividade de fumo e suscetibilidade da cultura em função do seu estádio de desenvolvimento. Engenharia Agrícola, v.27, n.spe, p.30-34, 2007b.

COSTA, A.G.F. et al. Efeitos de ponta de pulverização na deriva de glyphosate $+2,4-\mathrm{D}$ em condições de campo. Revista Brasileira de Herbicidas, v.11, n.1, p.62-70, 2012.

FLINT, J.L.; BARRETT, M. Effects of glyphosate combinations with 2,4-D or dicamba on field bindweed (Convolvulus arvensis). Weed Science, v.37, n.1, p.12-18, 1989.

GAZZIERO, D.L.P. et al. Herbicide alternatives for 2,4-D in no-till cropping systems. In: INTERNATIONAL WEED SCIENCE CONGRESS, 3, 2000, Foz do Iguaçu-Brasil. Abstracts. Corvallis: Weed Science Society, 2000. p. 134.

GILREATH, J.P.; CHASE, C.A.; LOCASCIO, S.J. Crop injury from sublethal rates of herbicide. III. Hortscience, v.36, n.4, p.677681, 2001.

HEMPHILL, D.D.Jr.; MONTGOMERY, M.L. Response of vegetable crops to sublethal application of 2,4-D. Weed Science, v.29, n.6, p.632-635, 1981. 
KISSMANN, K. G. Rumos e tendências da pesquisa em tecnologia de aplicação de agrotóxicos: A visão da indústria química. In:

SIMPÓSIO INTERNACIONAL DE TECNOLOGIA DE APLICAÇÃO DE AGROTÓXICOS, 2, 2001. Jundiaí. Anais, Jundiaí: Instituto Agronômico de Campinas, 2001.

NUYTTENS, D. et al. Experimental study of factors influencing the risk of drift from Field sprayers, Part 2: Spray application technique. Aspects Applied Biology, v.77, n.2, p.331339, 2006.

OLIVEIRA JR, R.S. et al. Efeito de subdoses de 2,4-D na produtividade de uva Itália e suscetibilidade da cultura em função do seu estádio de desenvolvimento. Engenharia Agrícola, v.27, n. spe., p. 35-40, 2007.

OLIVEIRA NETO, A.M. et al. Estratégias de manejo de inverno e verão visando o controle de Conyza bonariensis e Bidens pilosa. Planta Daninha, v.20, n.spe, p.1107-1116, 2010a.

OLIVEIRA NETO, A.M. et al. Manejo de Conyza bonariensis com glyphosate $+2,4-\mathrm{D}$ e amônio-glufosinate em função do estádio de desenvolvimento. Revista Brasileira de Herbicidas, v.9, n.3, p.73-80, 2010 b.

RODRIGUES, B.N.; ALMEIDA, F.S. Guia de herbicidas. 6 ed. Londrina: RODRIGUES, B.N.; ALMEIDA, F.S. (Eds), 2011. 694p.

THEBALDI et al. Efeito da adição de adjuvante na redução da deriva em pontas de pulverização tipo cone vazio. Revista Ciências Técnicas Agropecuárias, v.18, n.2, p.1-6, 2009.

TUFFI SANTOS, L. D. et al. Intoxicação de espécies de eucalipto submetidas à deriva de glyphosate. Planta Daninha, v. 24, n. 2, p. 359-364, 2006.

YAMASHITA, O. M. et al. Resposta de varjão (Parkia multijuga) a subdoses de glyphosate. Planta Daninha, v. 24, n. 3, p. 527-531, 2006.
YAMASHITA, O.M.; GUIMARÃES, S.C. Deriva simulada de glyphosate em algodoeiro: efeito de dose, cultivar e estádio de desenvolvimento. Planta Daninha, v.24, n.4, p. 821-826, 2006.

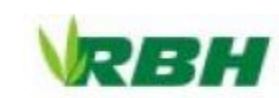

Rev. Bras. Herb., v.11, n.3, p.332-338, set./dez. 2012 